\title{
Economic analysis of residential PV self-consumption systems with Li-ion batteries under different billing scenarios
}

\author{
Carlos Galilea, Julio Pascual, Alberto Berrueta, Alfredo Ursua, Luis Marroyo \\ Department of Electrical, Electronic and Communication Engineering \\ Institute of Smart Cities (ISC) \\ Public University of Navarre (UPNA) \\ Pamplona, Spain \\ carlos.galilea@unavarra.es
}

\begin{abstract}
In this paper, an economic analysis for four houses with a PV self-consumption system with and without Li-ion batteries is carried out. In particular three different ways of sizing PV and batteries are analyzed under three different billing scenarios for the compensation of surplus energy injected into the grid. All methods run under the same energy strategy, which maximizes self-consumption. The three billing scenarios are: (1) no retribution for surplus energy, (2) retribution at pool price (net billing), and (3) monthly net metering. This study shows how fixed costs make these systems just profitable for small systems. Moreover, the results show how the battery cost and lifespan affects the final profitability of the system and what future evolution in these factors is needed for making these systems profitable under different billing methods.
\end{abstract}

Keywords-PV self-consumption, battery storage, netmetering, net-billing, distributed generation, prosumer, economic analysis

\section{INTRODUCTION}

There is an increasing concern worldwide to tackle global warming by different means, including the use of less fossil fuels by combining energy efficiency methods and integrating more renewable energies into the system. At the 2012 United Nations Climate Change Conference, in Doha, the Kyoto [1] protocol was extended until 2020 and later on, at the Paris Agreement [2], some specific goals were set being now put in practice by regions and countries involved. For instance, these ambitions are reflected in different governmental plans in Europe [3], [4] and Spain [5], [6], in order to have a more renewable energy system. These plans include the residential sector, changing the energy generation model to a more distributed one, by installing renewable energy sources in buildings, sometimes with energy storage and an energy management system (microgrids) [7]-[9]. These policies along with the continuous rise of electricity prices and the cost reduction of renewable energy costs, especially photovoltaic (PV) power, which has reach grid parity in many regions [10], has brought worldwide interest to final users to install such systems in their homes [11].

However, in Spain, due to Government opposition at first and later instability, there has been great uncertainty on the way self-consumption is to be regulated. This uncertainty is slowing down the adoption of self-consumption in the residential sector given that these systems constitute a long-term investment and whose optimal sizing depends critrically on the legal regulation.

Almacenamiento Renovable Avanzado de Litio para Autoconsumo Residencial interconectado (ARALAR) - 0011-1411-2017-000021, Proyecto de I+D Gobierno de Navarra, and Hibridación de tecnologías avanzadas de almacenamiento eléctrico para sistemas basados en energías renovables DPI2016-80642-R (HIBRITAER)
Several studies exist on this topic for former regulations in Spain [12], [13], and billing systems in European countries [14] including Spain [15], [16], with batteries [17] and considering net metering [18]. In this study, different sizing and regulations are compared under otherwise same conditions in order to understand the best sizing solutions for each case, stressing the importance of a stable regulation frame for the optimal design of these long-term systems. Furthermore, the key factors for the profitability of these systems are analyzed. Note that different consumption profiles are analyzed under different billing systems and sizing methods, permitting to understand their influence in profitability.

In particular, in Section II, the case studies, prices, sizing methods and billing methods are described. Moreover, the base cases, i.e., the houses without self-consumption, are analyzed in terms of electricity billing. In Section III, the results for the profitability of every system under all the sizing and billing methods are shown and analyzed. Finally, in section IV, the major conclusions are presented.

\section{Problem Statement}

\section{A. Case studies}

All four single-family homes analyzed (named A to D) are located in Pamplona (Spain). Hourly power consumption profiles from year 2017 are available for the analysis. The total consumption over the year of these homes ranges from 2980 to $5115 \mathrm{kWh}$, being around the national average in the residential sector [19]. The annual bill for these cases without the self-consumption system serves as the reference case against which the rest of the cases will be compared. Besides, the characteristics of the roofs are known which permits to calculate the PV power available during the year for every case.

\section{B. $P V$ and battery sizing}

The analysis has been carried out considering three PV and battery sizing options shown in Table I.

TABLE I. SIZING OPTIONS CONSIDERED FOR ANALYSIS

\begin{tabular}{|l|l|c|}
\hline & \multicolumn{1}{|c|}{$\begin{array}{c}\text { PV power } \\
{[\mathbf{k W}]}\end{array}$} & $\begin{array}{c}\text { Battery capacity } \\
{[\mathbf{k W h}]}\end{array}$ \\
\hline Sizing I & $\mathrm{E}_{\mathrm{PV} \text { year }} \equiv 120 \% \mathrm{E}_{\text {cons year }}$ & $80 \% \overline{\mathrm{E}}_{\text {cons day }}$ \\
\hline Sizing II & $\mathrm{E}_{\mathrm{PV} \text { year }} \equiv 90 \% \mathrm{E}_{\text {cons year }}$ & $20 \% \overline{\mathrm{E}}_{\text {cons day }}$ \\
\hline Sizing III & $\mathrm{E}_{\mathrm{PV} \text { year }} \equiv 120 \% \mathrm{E}_{\text {cons year }}$ & 0 \\
\hline
\end{tabular}


The first sizing method seeks to maximize selfconsumption by oversizing PV power so it generates $20 \%$ more energy than it is consumed over a year and installing a Li-ion battery that can store as much energy as $80 \%$ of the average daily energy consumption. This sizing is oriented to policies that do not compensate for energy surplus.

The second sizing method seeks a better self-consumption ratio than without a battery but trying to get a higher profitability than Sizing I. For this purpose, PV size has been reduced, but specially battery size. In particular, sizing is set so that generated PV power accounts for as much as $90 \%$ of energy consumption and battery has to be able to store $20 \%$ of the average daily energy consumption.

Finally, Sizing III has the same PV power as Sizing I but has no battery. This sizing is oriented to a scenario in which energy surplus is compensated.

In order to obtain the PV power needed according to the three proposed methods, PV generation has been calculated for every case in a ten minute basis over a year using radiation and temperature data from a weather station in Pamplona for the year 2018. This year had a horizontal radiation of $1419 \mathrm{kWh} / \mathrm{m}^{2}$, very close to that of the typical meteorological year, $1480 \mathrm{kWh} / \mathrm{m}^{2}$. Finally note that for the simulation, peak power of PV panels has been reduced by $5 \%$ in order to take into account degradation over time.

Regarding the power of the converter, in all cases, it has been chosen so that it can cope with the maximum power flow observed by simulation.

\section{Energy management strategy}

The typical electric system in a house with PV selfconsumption and battery comprises the elements seen in Fig. 1. The elements om the DC side are the PV panels and battery, which are connected to a hybrid converter, which includes converters for PV and batteries and a common inverter. The loads and grid are connected to the AC side of the inverter.

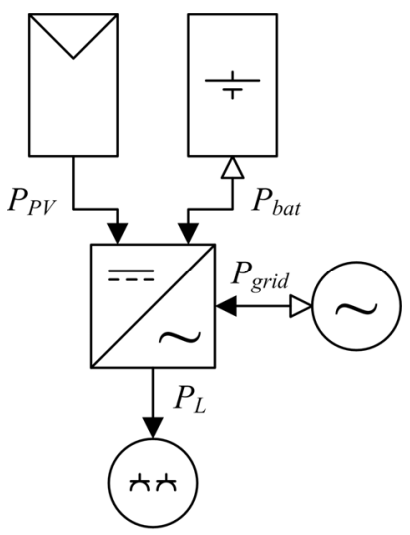

Fig. 1. Power flows in the self-consumption system. Black solid arrows indicate positive direction of bidirectional flows.

Furthermore, the power flows in the system are depicted in Fig. 1. While power from the PV panels $\left(P_{P V}\right)$ and to the loads $\left(P_{L}\right)$ is unidirectional, power from the battery $\left(P_{b a t}\right)$ and the grid $\left(P_{\text {grid }}\right)$ is bidirectional, in which case the positive direction is indicated with the black solid arrow. Neglecting power losses, at every moment the sum of these power flows is zero as shown in Eq. (1):

$$
P_{P V}+P_{b a t}+P_{\text {grid }}-P_{L}=0
$$

However, the amount of power from the grid or the battery can be changed. The energy strategy is in charge of deciding these values. In all cases, the energy strategy used is aimed at maximizing self-consumption, defined as the ratio between the energy income from the PV panels and battery divided by the energy consumed by the loads and the energy that the batteries may consume from the grid, which only happens whenever their SOC lies below a value of $3 \%$.

As an example, Fig. 2 shows the power profiles of one of the case during two days. In this graph, it can be seen how PV power is delivered to the loads and the battery only injecting into the grid when the battery is full. At night, the batteries provide the energy to the loads until they reach a SOC of 5\%, when the loads start consuming from the grid.

For the simulation, an efficiency of $95 \%$ has been used for all the power flows in the converter as well as for the charge and discharge of the battery.

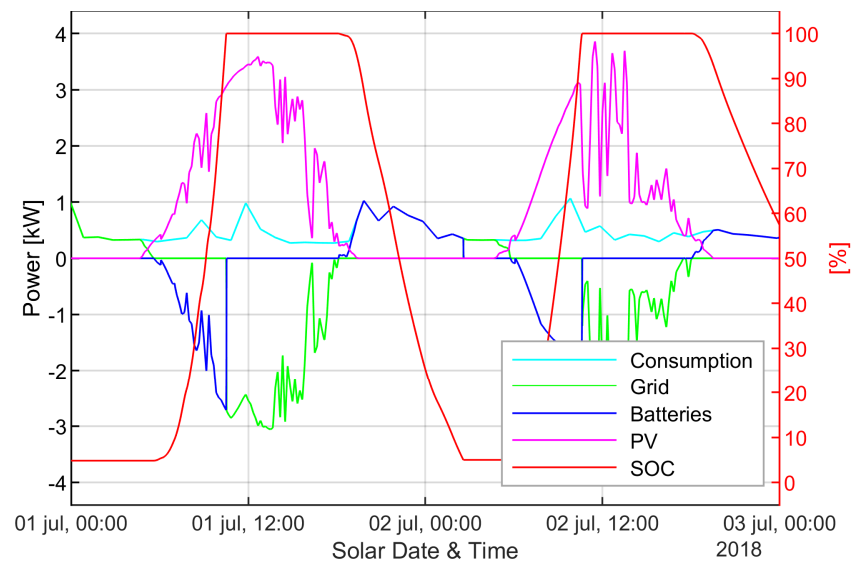

Fig. 2. Power profiles during two days for household D and sizing I.

\section{Costs and lifespan of equipment, fixtures and labour}

Prices from different manufacturers and installers has been collected and linearized as a function of PV peak power (in the case of PV panels, fixtures, labour and converter) and as a function of battery capacity for the battery. Typical lifespans have been used for every element as shown in (Table II). As it can be seen, fixed costs are quite high so lower profitability is expected for smaller systems.

TABLE II.

COSTS AND LIFESPAN OF MAIN ELEMENTS

\begin{tabular}{|l|l|c|}
\hline \multicolumn{1}{|c|}{$\begin{array}{c}\text { Cost } \\
{[€]}\end{array}$} & $\begin{array}{c}\text { Lifespan } \\
\text { [years] }\end{array}$ \\
\hline PV panels & Cost $_{P V}=423.50 P_{P V}\left(\mathrm{~kW}_{\mathrm{p}}\right)$ & 25 \\
\hline Converter & Cost $_{\text {Inv }}=121 P_{n}\left(\mathrm{~kW}_{\mathrm{n}}\right)+2178$ & 20 \\
\hline $\begin{array}{l}\text { Fixtures and } \\
\text { labour }\end{array}$ & Cost $_{\text {Inst }}=172.9 P_{P V}\left(\mathrm{~kW}_{\mathrm{p}}\right)+3630$ & 40 \\
\hline Battery & Cost $_{\text {Bat }}=382.8 C_{\text {Bat }}(\mathrm{kWh})+1688.9$ & 10 \\
\hline
\end{tabular}

\section{E. Energy billing}

For the four houses without a self-consumption system, the yearly energy bill has been calculated using the two most common tariffs in Spain for the residential sector (named 2.0A and 2.0DHA, and corresponding to single-rate and time-ofuse tariffs respectively). Although electricity prices in Spain can be established in the free market by electricity retailers, prices used for this study are those established by the Spanish 
Government, known as PVPC (Spanish initials for Small Consumer Voluntary Price). In particular, as prices are variable, the average during year 2018 has been used. These prices along with taxes and other charges applicable are shown in Table III.

TABLE III. BILLING COMPONENTS

\begin{tabular}{|c|c|c|c|c|c|c|}
\hline \multirow{2}{*}{ Tariff } & \multirow{2}{*}{$\begin{array}{c}\text { Contracted } \\
\text { power } \\
\text { price } \\
{[€ / \mathbf{k W} \text { year] }}\end{array}$} & \multicolumn{2}{|c|}{$\begin{array}{c}\text { Energy price } \\
{[€ / \mathbf{k W h}]}\end{array}$} & \multirow{2}{*}{$\begin{array}{c}\text { Electricity } \\
\operatorname{tax} \\
{[\%]}\end{array}$} & \multirow{2}{*}{$\begin{array}{c}\text { Energy } \\
\text { meter cost } \\
\text { [€/month] }\end{array}$} & \multirow{2}{*}{$\begin{array}{l}\text { VAT } \\
{[\%]}\end{array}$} \\
\hline & & $\begin{array}{c}\text { Peak } \\
\text { (P1) }\end{array}$ & $\begin{array}{c}\text { Off-peak } \\
(P 2)\end{array}$ & & & \\
\hline $2.0 \mathrm{~A}$ & 42.043426 & \multicolumn{2}{|c|}{0.124677} & & & \\
\hline 2.0DHA & 42.043426 & 0.145274 & 0.072426 & & & \\
\hline
\end{tabular}

In Table IV, the yearly bills using prices in Table III and the power consumptions of every home are shown. For all cases, a contracted power of $4.6 \mathrm{~kW}$ has been used. As it can be seen, in all cases tariff 2.0DHA results in the lower bill so this will be the only tariff considered from now on in this study. Moreover, this tariff should be the most appropriate for PV self-consumption as PV generation occurs precisely during peak hours.

TABLE IV. YEARLY BILLS FOR BASE CASES

\begin{tabular}{|c|c|c|c|c|c|}
\hline \multirow[t]{2}{*}{ House } & \multirow[t]{2}{*}{ Tariff } & \multirow{2}{*}{$\begin{array}{c}\text { Contracted } \\
\text { power } \\
{[\mathrm{kW}]}\end{array}$} & \multicolumn{2}{|c|}{$\begin{array}{c}\text { Yearly energy } \\
{[\mathrm{kWh}]}\end{array}$} & \multirow{2}{*}{$\begin{array}{c}\text { Yearly } \\
\text { electricity } \\
\text { bill } \\
{[€]}\end{array}$} \\
\hline & & & $P 1$ & $P 2$ & \\
\hline \multirow{2}{*}{$\mathbf{A}$} & $2.0 \mathrm{~A}$ & \multirow{2}{*}{4.6} & \multicolumn{2}{|c|}{2980.1} & 730.30 \\
\hline & 2.0DHA & & 1524.7 & 1455.4 & 673.52 \\
\hline \multirow{2}{*}{ B } & $2.0 \mathrm{~A}$ & \multirow{2}{*}{4.6} & \multicolumn{2}{|c|}{3770.2} & 855.60 \\
\hline & 2.0DHA & & 1767.0 & 2003.2 & 768.76 \\
\hline \multirow{2}{*}{ C } & $2.0 \mathrm{~A}$ & \multirow{2}{*}{4.6} & \multicolumn{2}{|c|}{4279.2} & 936.30 \\
\hline & 2.0DHA & & 1910.4 & 2368.8 & 828.92 \\
\hline \multirow{2}{*}{ D } & $2.0 \mathrm{~A}$ & \multirow{2}{*}{4.6} & \multicolumn{2}{|c|}{5115.1} & 1068.80 \\
\hline & 2.0DHA & & 2575.1 & 2540.0 & 967.52 \\
\hline
\end{tabular}

Regarding the contracted power for the self-consumption scenarios, it will be considered as the maximum power exchanged with the grid as calculated in the simulations. Moreover, for the self-consumption cases, three different scenarios are considered regarding the compensation for the energy injected into the grid:

1) No compensation: In this scenario, the savings will only come from the self-consumed energy, i.e., from the energy not consumed from the grid. Note that anyway, net metering has been applied in an hourly basis, as this is the case in Spain, where billing is applied per hour.

2) Net billing: in this case, the energy fed into the grid is sold at pool price as stated in RD244/2019 [20]. However, it can only compensate for the amount of money that accounts for the energy bought before taxes.

3) Net-metering: in this third case, the energy injected is compensated by the energy absorbed on a monthly basis.

\section{SIMULATION RESULTS AND ECONOMIC ANALYSIS}

All cases are simulated using the maximum selfconsumption strategy. As a result, grid power profiles are obtainned which allow calculating the yearly energy bills for every house, sizing and billing method, as shown in Table V. Comparing these results with the base cases in Table IV, the yearly savings can be obtained as also shown in Table V. In all cases, savings in the energy bill are notably high, ranging from $40 \%$ up to $70 \%$.

However, in order for the project to be profitable, the savings obtained must offset the cost of the self-consumption system. In order to calculate that, the cost per element has been annualized by dividing it by its lifespan. By adding every annualized cost, the total annualized cost can be calculated. Finally, the annual benefit can be calculated, subtracting the annualized cost to the yearly savings (Table VI).

Moreover, the years in which the savings must have paid off the self-consumption system can be calculated by dividing the total cost by the annualized cost. If the payback period is lower than this value, then the project is viable. This provides not only a way to check the profitability of the project, but also its payback period, which in case of being too high, in spite of being profitable, may discourage some investors. As it can be seen in Table V, not all projects are profitable as profitability depends on sizing and billing method.

In particular, regarding Sizing I, having the highest PV peak power and energy storage capacity, it draws the highest self-consumption rates (around 68\%) and, as such, the highest savings in electricity bills. Given that this solution provides the highest rate of self-consumption, one would expect it to be the best solution for Scenario I, however, the high price and low lifespan of the battery results in negative overall benefit. Note that, in this case, around $63 \%$ of the total annualized cost corresponds to the battery. Nevertheless, it has been calculated that at an average price of $428 € / \mathrm{kWh}$ and with a lifespan of 15 years, this system would be profitable in houses A, B and $\mathrm{D}$, for all billing methods. In the case of house $\mathrm{C}$, the reduction in average price should be even higher $(370 € / \mathrm{kWh})$ as, despite not having such a low consumption as house $\mathrm{A}$, its power profile is lower during sun hours and higher during mornings and night.

Sizing II, with a notably lower investment in batteries, allows for a lower self-consumption ratio, around $45 \%$. However, although annual profitability improves in all billing methods, it is only profitable in house $\mathrm{D}$ (highest consumption case) for billing methods II and III.

Finally, regarding Sizing III, it gets the best results in all billing scenarios and houses. In this case, self-consumption lies around $35 \%$ so reduction in energy bill is not high. However, the lack of investment in batteries makes it the most profitable case. In particular, this sizing along with billing method 3, gives the optimal results, given that net metering is more profitable than storing in a battery at given prices.

Finally, note that at commercial prices, some of these projects are highly profitable and others are close to be profitable. The decreasing costs of batteries and its raising lifespan is the key factor to make these projects more profitable in the near future. 
TABLE V. YEARLY BILLING FOR THE SELF-CONSUMPTION CASES FOR EVERY HOME, SIZING AND BILLING SCENARIO

\begin{tabular}{|c|c|c|c|c|c|c|c|c|c|c|c|c|c|c|c|}
\hline \multirow{2}{*}{\multicolumn{2}{|c|}{$\begin{array}{c}\text { House } \\
\text { A }\end{array}$}} & \multirow{2}{*}{$\begin{array}{c}\text { PV } \\
\text { Power } \\
{\left[\mathbf{k W}_{\mathrm{p}}\right]}\end{array}$} & \multirow{2}{*}{$\begin{array}{c}\text { Battery } \\
\text { size } \\
{[\mathrm{kWh}]}\end{array}$} & \multirow{2}{*}{\begin{tabular}{|c|}
$\begin{array}{c}\text { Contracted } \\
\text { power } \\
{[\mathrm{kW}]}\end{array}$ \\
\end{tabular}} & \multirow{2}{*}{$\begin{array}{c}\text { Power } \\
\text { billing } \\
{[€]}\end{array}$} & \multirow{2}{*}{$\begin{array}{c}\text { PV yearly } \\
\text { energy } \\
{[\mathrm{kWh}]}\end{array}$} & \multirow{2}{*}{$\begin{array}{c}\text { Self-consumed } \\
\text { energy } \\
{[\mathrm{kWh}]}\end{array}$} & \multicolumn{2}{|c|}{$\begin{array}{c}\text { Energy from grid } \\
{[\mathrm{kWh}]}\end{array}$} & \multicolumn{2}{|c|}{$\begin{array}{c}\text { Energy to grid } \\
{[\mathrm{kWh}]}\end{array}$} & \multicolumn{2}{|c|}{$\begin{array}{c}\text { Energy billing } \\
\text { [€/year] }\end{array}$} & \multirow{2}{*}{$\begin{array}{c}\begin{array}{c}\text { Total } \\
\text { billing } \\
\text { [€/year] }\end{array} \\
238.02 \\
\end{array}$} & \multirow{2}{*}{$\begin{array}{c}\begin{array}{c}\text { Yearly } \\
\text { savings } \\
\text { [ } € / \text { year] }\end{array} \\
435.50\end{array}$} \\
\hline & & & & & & & & & & & & & & & \\
\hline \multirow{3}{*}{$\begin{array}{l}\text { Siz. } \\
\text { I }\end{array}$} & Bill. 1 & \multirow{3}{*}{2.6} & \multirow{3}{*}{6.5} & \multirow{3}{*}{2.2} & \multirow{3}{*}{92.50} & \multirow{3}{*}{3539.7} & \multirow{3}{*}{2128.2} & \multirow{3}{*}{325.4} & \multirow{3}{*}{526.5} & \multirow{3}{*}{1110.6} & \multirow{3}{*}{80.7} & 47.27 & 38.13 & & \\
\hline & Bill. 2 & & & & & & & & & & & 40.79 & 36.69 & 227.95 & 445.57 \\
\hline & Bill. 3 & & & & & & & & & & & 35.23 & 36.46 & 220.58 & 452.94 \\
\hline \multirow{3}{*}{$\begin{array}{l}\text { Siz. } \\
\text { II }\end{array}$} & Bill. 1 & \multirow{3}{*}{2.0} & \multirow{3}{*}{1.6} & \multirow{3}{*}{1.7} & \multirow{3}{*}{71.47} & \multirow{3}{*}{2722.8} & \multirow{3}{*}{1471.2} & \multirow{3}{*}{515.6} & \multirow{3}{*}{993.3} & \multirow{3}{*}{1049.7} & \multirow{3}{*}{109.94} & 74.90 & 71.94 & 289.44 & 384.08 \\
\hline & Bill. 2 & & & & & & & & & & & 56.34 & 65.35 & 257.44 & 416.08 \\
\hline & Bill. 3 & & & & & & & & & & & 40.82 & 63.98 & 235.96 & 437.56 \\
\hline \multirow{3}{*}{$\begin{array}{l}\text { Siz. } \\
\text { III }\end{array}$} & Bill. 1 & \multirow{3}{*}{2.6} & & & & & & & & & & 110.91 & 73.55 & 364.02 & 309.50 \\
\hline & Bill. 2 & & 0.0 & 2.2 & 92.50 & 3539.7 & 1201.0 & 763.5 & 1015.6 & 1704.4 & 634.2 & 65.76 & 40.25 & 264.24 & 409.28 \\
\hline & Bill. 3 & & & & & & & & & & & 28.42 & 36.15 & 211.52 & 462.00 \\
\hline
\end{tabular}

\begin{tabular}{|c|c|c|c|c|c|c|c|c|c|c|c|c|c|c|c|}
\hline \multirow{2}{*}{\multicolumn{2}{|c|}{$\begin{array}{c}\text { House } \\
\text { B }\end{array}$}} & \multirow{2}{*}{$\begin{array}{c}\text { PV } \\
\text { Power } \\
{\left[\mathrm{kW}_{\mathrm{p}}\right]}\end{array}$} & \multirow{2}{*}{$\begin{array}{c}\text { Battery } \\
\text { size } \\
{[\mathbf{k W h}]}\end{array}$} & \multirow{2}{*}{\begin{tabular}{|c|}
$\begin{array}{c}\text { Contracted } \\
\text { power } \\
{[\mathrm{kW}]}\end{array}$ \\
\end{tabular}} & \multirow{2}{*}{$\begin{array}{c}\text { Power } \\
\text { billing } \\
{[€]}\end{array}$} & \multirow{2}{*}{$\begin{array}{l}\text { PV yearly } \\
\text { energy } \\
{[\mathrm{kWh}]}\end{array}$} & \multirow{2}{*}{$\begin{array}{c}\text { Self-consumed } \\
\text { energy } \\
{[\mathrm{kWh}]}\end{array}$} & \multicolumn{2}{|c|}{$\begin{array}{l}\text { Energy from grid } \\
{[\mathrm{kWh}]}\end{array}$} & \multicolumn{2}{|c|}{$\begin{array}{c}\text { Energy to grid } \\
{[\mathrm{kWh}]}\end{array}$} & \multicolumn{2}{|c|}{$\begin{array}{c}\text { Energy billing } \\
\text { [€/year] }\end{array}$} & \multirow{2}{*}{$\begin{array}{c}\text { Total } \\
\text { billing } \\
\text { [€/year] }\end{array}$} & \multirow{2}{*}{$\begin{array}{l}\text { Yearly } \\
\text { savings } \\
\text { [E/year] }\end{array}$} \\
\hline & & & & & & & & $P 1$ & $P 2$ & P1 & $P 2$ & $P 1$ & $P 2$ & & \\
\hline \multirow{3}{*}{$\begin{array}{c}\text { Siz. } \\
\text { I }\end{array}$} & Bill. 1 & \multirow{3}{*}{3.3} & \multirow{3}{*}{8.3} & \multirow{3}{*}{2.6} & \multirow{3}{*}{109.31} & \multirow{3}{*}{4492.7} & \multirow{3}{*}{2895.0} & \multirow{3}{*}{249.6} & \multirow{3}{*}{625.6} & \multirow{3}{*}{1221.2} & \multirow{3}{*}{67.3} & 36.27 & 45.31 & 254.55 & 514.21 \\
\hline & Bill. 2 & & & & & & & & & & & 29.86 & 44.06 & 244.82 & 523.94 \\
\hline & Bill. 3 & & & & & & & & & & & 23.79 & 43.87 & 236.85 & 531.91 \\
\hline \multirow{3}{*}{$\begin{array}{l}\text { Siz. } \\
\text { II }\end{array}$} & Bill. 1 & \multirow{3}{*}{2.5} & \multirow{3}{*}{2.1} & \multirow{3}{*}{1.9} & \multirow{3}{*}{79.88} & \multirow{3}{*}{3403.6} & \multirow{3}{*}{1982.4} & \multirow{3}{*}{449.8} & \multirow{3}{*}{1338.0} & \multirow{3}{*}{1226.1} & \multirow{3}{*}{72.6} & 65.35 & 96.910 & 319.73 & 449.03 \\
\hline & Bill. 2 & & & & & & & & & & & 45.78 & 92.55 & 289.30 & 479.46 \\
\hline & Bill. 3 & & & & & & & & & & & 28.15 & 91.65 & 265.73 & 503.04 \\
\hline \multirow{3}{*}{$\begin{array}{l}\text { Siz. } \\
\text { III }\end{array}$} & Bill. 1 & \multirow{3}{*}{3.3} & & & & & & & & & & 116.15 & 98.22 & 428.79 & 339.97 \\
\hline & Bill. 2 & & 0.0 & 2.7 & 113.52 & 4492.7 & 1614.5 & 799.5 & 1356.2 & 2162.0 & 716.2 & 56.89 & 58.36 & 302.72 & 466.04 \\
\hline & Bill. 3 & & & & & & & & & & & 9.70 & 52.12 & 234.77 & 533.99 \\
\hline
\end{tabular}

\begin{tabular}{|c|c|c|c|c|c|c|c|c|c|c|c|c|c|c|c|}
\hline \multirow{2}{*}{\multicolumn{2}{|c|}{$\begin{array}{c}\text { House } \\
\text { C }\end{array}$}} & \multirow{2}{*}{$\begin{array}{c}\text { PV } \\
\text { Power } \\
{\left[\mathbf{k W} W_{\mathrm{p}}\right]}\end{array}$} & \multirow{2}{*}{$\begin{array}{c}\text { Battery } \\
\text { size } \\
{[\mathbf{k W h}]}\end{array}$} & \multirow{2}{*}{\begin{tabular}{|c|}
$\begin{array}{c}\text { Contracted } \\
\text { power } \\
{[\mathrm{kW}]}\end{array}$ \\
\end{tabular}} & \multirow{2}{*}{$\begin{array}{c}\text { Power } \\
\text { billing } \\
{[€]}\end{array}$} & \multirow{2}{*}{$\begin{array}{c}\text { PV yearly } \\
\text { energy } \\
\text { [kWh] }\end{array}$} & \begin{tabular}{|c|} 
Self-consumed \\
energy \\
{$[\mathrm{kWh}]$}
\end{tabular} & \multicolumn{2}{|c|}{$\begin{array}{c}\text { Energy from grid } \\
{[\mathrm{kWh}]}\end{array}$} & \multicolumn{2}{|c|}{$\begin{array}{l}\text { Energy to grid } \\
{[\mathrm{kWh}]}\end{array}$} & \multicolumn{2}{|c|}{$\begin{array}{c}\text { Energy billing } \\
\text { [€/year] }\end{array}$} & $\begin{array}{c}\text { Total } \\
\text { billing } \\
\text { [€/year] }\end{array}$ & $\begin{array}{l}\text { Yearly } \\
\text { savings } \\
\text { [€/year] }\end{array}$ \\
\hline & & & & & & & & & & & & & & & \\
\hline \multirow{3}{*}{$\begin{array}{l}\text { Siz. } \\
\text { I }\end{array}$} & Bill. 1 & \multirow{3}{*}{3.8} & \multirow{3}{*}{9.4} & \multirow{3}{*}{2.9} & \multirow{3}{*}{121.93} & \multirow{3}{*}{5169.5} & \multirow{3}{*}{3075.3} & \multirow{3}{*}{390.3} & \multirow{3}{*}{813.6} & \multirow{3}{*}{1519.2} & \multirow{3}{*}{202.7} & 56.70 & 58.93 & 313.90 & 515.02 \\
\hline & Bill. 2 & & & & & & & & & & & 48.03 & 56.34 & 299.58 & 529.34 \\
\hline & Bill. 3 & & & & & & & & & & & 41.00 & 56.05 & 290.27 & 538.65 \\
\hline \multirow{3}{*}{$\begin{array}{l}\text { Siz. } \\
\text { II }\end{array}$} & Bill. 1 & \multirow{3}{*}{2.8} & \multirow{3}{*}{2.3} & \multirow{3}{*}{2.4} & \multirow{3}{*}{100.90} & \multirow{3}{*}{3809.1} & \multirow{3}{*}{2091.1} & \multirow{3}{*}{610.2} & \multirow{3}{*}{1577.9} & \multirow{3}{*}{1375.6} & \multirow{3}{*}{207.1} & 88.65 & 114.28 & 398.20 & 430.73 \\
\hline & Bill. 2 & & & & & & & & & & & 65.66 & 101.86 & 353.16 & 475.76 \\
\hline & Bill. 3 & & & & & & & & & & & 46.89 & 99.28 & 326.01 & 502.91 \\
\hline \multirow{3}{*}{$\begin{array}{l}\text { Siz. } \\
\text { III }\end{array}$} & Bill. 1 & \multirow{3}{*}{3.8} & & & & & & & & & & 143.42 & 117.24 & 498.36 & 330.56 \\
\hline & Bill. 2 & & 0.0 & 2.9 & 121.93 & 5169.5 & 1673.2 & 987.3 & 1618.7 & 2341.4 & 1086.1 & 81.22 & 63.89 & 351.39 & 477.53 \\
\hline & Bill. 3 & & & & & & & & & & & 30.74 & 57.96 & 279.66 & 549.26 \\
\hline
\end{tabular}

\begin{tabular}{|c|c|c|c|c|c|c|c|c|c|c|c|c|c|c|c|}
\hline \multirow{2}{*}{\multicolumn{2}{|c|}{$\begin{array}{c}\text { House } \\
\text { D }\end{array}$}} & \multirow{2}{*}{$\begin{array}{c}\text { PV } \\
\text { Power } \\
{\left[\mathbf{k W}_{\mathrm{p}}\right]}\end{array}$} & \multirow{2}{*}{$\begin{array}{c}\text { Battery } \\
\text { size } \\
{[\mathbf{k W h}]}\end{array}$} & \multirow{2}{*}{\begin{tabular}{|c|}
$\begin{array}{c}\text { Contracted } \\
\text { power } \\
{[\mathrm{kW}]}\end{array}$ \\
\end{tabular}} & \multirow{2}{*}{$\begin{array}{c}\text { Power } \\
\text { billing } \\
{[€]}\end{array}$} & \multirow{2}{*}{$\begin{array}{l}\text { PV yearly } \\
\text { energy } \\
\text { [kWh] }\end{array}$} & \multirow{2}{*}{$\begin{array}{c}\text { Self-consumed } \\
\text { energy } \\
{[\mathrm{kWh}]}\end{array}$} & \multicolumn{2}{|c|}{$\begin{array}{c}\text { Energy from grid } \\
{[\mathrm{kWh}]}\end{array}$} & \multicolumn{2}{|c|}{$\begin{array}{c}\text { Energy to grid } \\
{[\mathrm{kWh}]}\end{array}$} & \multicolumn{2}{|c|}{$\begin{array}{c}\text { Energy billing } \\
\text { [€/year] }\end{array}$} & \multirow{2}{*}{$\begin{array}{c}\text { Total } \\
\text { billing } \\
\text { [€/year] }\end{array}$} & \multirow{2}{*}{$\begin{array}{l}\text { Yearly } \\
\text { savings } \\
\text { [€/year] }\end{array}$} \\
\hline & & & & & & & & $P 1$ & $P 2$ & $P 1$ & $P 2$ & $P 1$ & $P 2$ & & \\
\hline \multirow{3}{*}{ Siz. } & Bill. 1 & \multirow{3}{*}{4.5} & \multirow{3}{*}{11.2} & \multirow{3}{*}{3.5} & \multirow{3}{*}{147.15} & \multirow{3}{*}{6126.4} & \multirow{3}{*}{3965.0} & \multirow{3}{*}{419.4} & \multirow{3}{*}{730.8} & \multirow{3}{*}{1668.9} & \multirow{3}{*}{103.4} & 60.92 & 52.93 & 343.72 & 623.80 \\
\hline & Bill. 2 & & & & & & & & & & & 48.05 & 50.89 & 324.76 & 642.75 \\
\hline & Bill. 3 & & & & & & & & & & & 36.98 & 50.65 & 310.38 & 657.14 \\
\hline \multirow{3}{*}{$\begin{array}{l}\text { Siz. } \\
\text { II }\end{array}$} & Bill. 1 & \multirow{3}{*}{3.4} & \multirow{3}{*}{2.8} & \multirow{3}{*}{2.6} & \multirow{3}{*}{109.31} & \multirow{3}{*}{4628.8} & \multirow{3}{*}{2800.1} & \multirow{3}{*}{681.6} & \multirow{3}{*}{1633.4} & \multirow{3}{*}{1556.2} & \multirow{3}{*}{115.1} & 99.02 & 118.30 & 427.19 & 540.32 \\
\hline & Bill. 2 & & & & & & & & & & & 71.10 & 111.40 & 382.90 & 584.62 \\
\hline & Bill. 3 & & & & & & & & & & & 46.69 & 110.40 & 350.60 & 616.92 \\
\hline \multirow{3}{*}{$\begin{array}{l}\text { Siz. } \\
\text { III }\end{array}$} & Bill. 1 & \multirow{3}{*}{4.5} & & & & & & & & & & 157.63 & 119.97 & 557.33 & 410.18 \\
\hline & Bill. 2 & & 0.0 & 3.6 & 151.36 & 6126.4 & 2373.6 & 1085.1 & 1656.4 & 2777.4 & 975.3 & 83.91 & 66.96 & 396.15 & 571.37 \\
\hline & Bill. 3 & & & & & & & & & & & 21.79 & 57.62 & 305.26 & 662.25 \\
\hline
\end{tabular}


TABLE VI. GLOBAL YEARLY SAVINGS INSTALLATIONS ACCORDING TO SIZE AND STUDY SCENARIOS (IN GREEN, POSITIVE SAVINGS)

\begin{tabular}{|c|c|c|c|c|c|c|c|c|c|c|c|c|c|}
\hline \multicolumn{2}{|c|}{$\begin{array}{c}\text { House } \\
\text { A }\end{array}$} & $\begin{array}{c}\text { PV } \\
\text { Power } \\
{\left[\mathbf{k W}_{\mathrm{p}}\right]}\end{array}$ & $\begin{array}{c}\text { Battery } \\
\text { size } \\
{[\mathbf{k W h}]}\end{array}$ & $\begin{array}{c}\text { PV panels } \\
\text { cost } \\
{[€]}\end{array}$ & $\begin{array}{c}\text { Converter } \\
\text { cost } \\
{[€]}\end{array}$ & $\begin{array}{c}\text { Fixtures } \\
\text { and labour } \\
{[\epsilon]}\end{array}$ & $\begin{array}{c}\text { Battery } \\
\text { cost } \\
{[€]}\end{array}$ & $\begin{array}{c}\text { Total } \\
\text { Costs } \\
{[€]}\end{array}$ & $\begin{array}{c}\text { Annualized } \\
\text { total costs } \\
\text { [€/year] }\end{array}$ & $\begin{array}{c}\text { Weighed } \\
\text { Lifespa } \\
\text { [years] }\end{array}$ & $\begin{array}{l}\text { Yearly } \\
\text { Savings } \\
\text { [€/year] }\end{array}$ & $\begin{array}{c}\text { Payback } \\
\text { period } \\
\text { [years] }\end{array}$ & $\begin{array}{c}\text { Global } \\
\text { yearly } \\
\text { savings }[€]\end{array}$ \\
\hline \multirow{3}{*}{$\begin{array}{c}\text { Siz. } \\
\text { I }\end{array}$} & Bill. 1 & \multirow{3}{*}{2.6} & \multirow{3}{*}{6.5} & \multirow{3}{*}{1101.10} & \multirow{3}{*}{2405.40} & \multirow{3}{*}{4079.40} & \multirow{3}{*}{4176.30} & \multirow{3}{*}{11762.00} & \multirow{3}{*}{683.93} & \multirow{3}{*}{17.2} & 435.50 & 27.0 & -248.43 \\
\hline & Bill. 2 & & & & & & & & & & 445.57 & 26.4 & -238.36 \\
\hline & Bill. 3 & & & & & & & & & & 452.94 & 26.0 & -230.99 \\
\hline \multirow{3}{*}{$\begin{array}{c}\text { Siz. } \\
\text { II }\end{array}$} & Bill. 1 & \multirow{3}{*}{2.0} & \multirow{3}{*}{1.6} & \multirow{3}{*}{847.00} & \multirow{3}{*}{2355.80} & \multirow{3}{*}{3975.70} & \multirow{3}{*}{2300.50} & \multirow{3}{*}{9479.00} & \multirow{3}{*}{481.11} & \multirow{3}{*}{19.7} & 384.08 & 24.7 & -97.02 \\
\hline & Bill. 2 & & & & & & & & & & 416.08 & 22.8 & -65.02 \\
\hline & Bill. 3 & & & & & & & & & & 437.56 & 21.7 & -43.55 \\
\hline \multirow{3}{*}{$\begin{array}{l}\text { Siz. } \\
\text { III }\end{array}$} & Bill. 1 & \multirow{3}{*}{2.6} & \multirow{3}{*}{0.0} & \multirow{3}{*}{1101.10} & \multirow{3}{*}{2411.10} & \multirow{3}{*}{4079.40} & \multirow{3}{*}{0.00} & \multirow{3}{*}{7591.60} & \multirow{3}{*}{266.58} & \multirow{3}{*}{28.5} & 309.50 & 24.5 & 42.92 \\
\hline & Bill. 2 & & & & & & & & & & 409.28 & 18.5 & 142.70 \\
\hline & Bill. 3 & & & & & & & & & & 462.00 & 16.4 & 195.42 \\
\hline \multicolumn{2}{|c|}{$\begin{array}{c}\text { House } \\
\text { B }\end{array}$} & $\begin{array}{c}\text { PV } \\
\text { Power } \\
{[\mathbf{k W} \text { ] }}\end{array}$ & $\begin{array}{c}\text { Battery } \\
\text { size } \\
{[\mathrm{kWh}]}\end{array}$ & $\begin{array}{c}\text { PV panels } \\
\text { cost } \\
{[€]}\end{array}$ & $\begin{array}{c}\text { Converter } \\
\text { cost } \\
{[€]}\end{array}$ & $\begin{array}{c}\text { Fixtures } \\
\text { and labour } \\
{[€]}\end{array}$ & $\begin{array}{c}\text { Battery } \\
\text { cost } \\
{[€]}\end{array}$ & $\begin{array}{c}\text { Total } \\
\text { Costs } \\
{[€]}\end{array}$ & $\begin{array}{c}\text { Annualized } \\
\text { total costs } \\
\text { [€/year] }\end{array}$ & $\begin{array}{l}\text { Weighed } \\
\text { Lifespa } \\
\text { [years] }\end{array}$ & $\begin{array}{l}\text { Yearly } \\
\text { Savings } \\
\text { [€/year] }\end{array}$ & $\begin{array}{c}\text { Payback } \\
\text { period } \\
\text { [years] }\end{array}$ & $\begin{array}{c}\text { Global } \\
\text { yearly } \\
\text { savings }[€]\end{array}$ \\
\hline & Bill. 1 & & & & & & & & & & 514.21 & 25.1 & -255.81 \\
\hline $\begin{array}{c}\text { Siz. } \\
\text { I }\end{array}$ & Bill. 2 & 3.3 & 8.3 & 1397.50 & 2451.40 & 4200.40 & 4865.40 & 12915.00 & 770.02 & 16.8 & 523.94 & 24.6 & -246.07 \\
\hline & Bill. 3 & & & & & & & & & & 531.91 & 24.3 & -238.10 \\
\hline & Bill. 1 & & & & & & & & & & 449.03 & 22.3 & -63.15 \\
\hline $\begin{array}{l}\text { Siz. } \\
\text { II }\end{array}$ & Bill. 2 & 2.5 & 2.1 & 1058.80 & 2381.70 & 4062.20 & 2491.90 & 9994.50 & 512.18 & 19.5 & 479.46 & 20.8 & -32.72 \\
\hline & Bill. 3 & & & & & & & & & & 503.04 & 19.9 & -9.14 \\
\hline & Bill. 1 & & & & & & & & & & 339.97 & 23.7 & 56.11 \\
\hline $\begin{array}{l}\text { Siz. } \\
\text { III }\end{array}$ & Bill. 2 & 3.3 & 0.0 & 1397.50 & 2458.90 & 4200.40 & 0.00 & 8056.90 & 283.86 & 28.4 & 466.04 & 17.3 & 182.18 \\
\hline
\end{tabular}

\begin{tabular}{|c|c|c|c|c|c|c|c|c|c|c|c|c|c|}
\hline \multicolumn{2}{|c|}{$\begin{array}{c}\text { House } \\
\text { C }\end{array}$} & $\begin{array}{c}\text { PV } \\
\text { Power } \\
{\left[\mathbf{k W}_{\mathrm{p}}\right]}\end{array}$ & $\begin{array}{c}\text { Battery } \\
\text { size } \\
{[\mathbf{k W h}]}\end{array}$ & $\begin{array}{c}\text { PV panels } \\
\text { cost } \\
{[€]}\end{array}$ & $\begin{array}{c}\text { Converter } \\
\text { cost } \\
{[€]}\end{array}$ & $\begin{array}{c}\text { Fixtures } \\
\text { and labour } \\
{[€]}\end{array}$ & $\begin{array}{c}\text { Battery } \\
\text { cost } \\
{[€]}\end{array}$ & $\begin{array}{c}\text { Total } \\
\text { Costs } \\
{[€]}\end{array}$ & $\begin{array}{c}\text { Annualized } \\
\text { total costs } \\
\text { [€/year] }\end{array}$ & $\begin{array}{l}\text { Weighed } \\
\text { Lifespa } \\
\text { [years] }\end{array}$ & $\begin{array}{l}\text { Yearly } \\
\text { Savings } \\
\text { [€/year] }\end{array}$ & $\begin{array}{c}\text { Payback } \\
\text { period } \\
\text { [years] }\end{array}$ & $\begin{array}{c}\text { Global } \\
\text { yearly } \\
\text { savings }[€]\end{array}$ \\
\hline \multirow{3}{*}{$\begin{array}{c}\text { Siz. } \\
\text { I }\end{array}$} & Bill. 1 & \multirow{3}{*}{3.8} & \multirow{3}{*}{9.4} & \multirow{3}{*}{1609.30} & \multirow{3}{*}{2480.50} & \multirow{3}{*}{4286.90} & \multirow{3}{*}{5286.50} & \multirow{3}{*}{13663.00} & \multirow{3}{*}{824.21} & \multirow{3}{*}{16.6} & 515.02 & 26.5 & -309.19 \\
\hline & Bill. 2 & & & & & & & & & & 529.34 & 25.8 & -294.87 \\
\hline & Bill. 3 & & & & & & & & & & 538.65 & 25.4 & -285.56 \\
\hline \multirow{3}{*}{$\begin{array}{c}\text { Siz. } \\
\text { II }\end{array}$} & Bill. 1 & \multirow{3}{*}{2.8} & \multirow{3}{*}{2.3} & \multirow{3}{*}{1185.80} & \multirow{3}{*}{2428.90} & \multirow{3}{*}{4114.00} & \multirow{3}{*}{2568.40} & \multirow{3}{*}{10297.00} & \multirow{3}{*}{528.57} & \multirow{3}{*}{19.5} & 430.73 & 23.9 & -97.85 \\
\hline & Bill. 2 & & & & & & & & & & 475.76 & 21.6 & -52.81 \\
\hline & Bill. 3 & & & & & & & & & & 502.91 & 20.5 & -25.66 \\
\hline \multirow{3}{*}{$\begin{array}{l}\text { Siz. } \\
\text { III }\end{array}$} & Bill. 1 & \multirow{3}{*}{3.8} & \multirow{3}{*}{0.0} & \multirow{3}{*}{1609.30} & \multirow{3}{*}{2480.50} & \multirow{3}{*}{4286.90} & \multirow{3}{*}{0.00} & \multirow{3}{*}{8376.70} & \multirow{3}{*}{295.57} & \multirow{3}{*}{28.3} & 330.56 & 25.3 & 34.99 \\
\hline & Bill. 2 & & & & & & & & & & 477.53 & 15.5 & 181.96 \\
\hline & Bill. 3 & & & & & & & & & & 549.26 & 15.3 & 253.70 \\
\hline
\end{tabular}

\begin{tabular}{|c|c|c|c|c|c|c|c|c|c|c|c|c|c|}
\hline \multicolumn{2}{|c|}{$\begin{array}{c}\text { House } \\
\text { D }\end{array}$} & $\begin{array}{c}\text { PV } \\
\text { Power } \\
{\left[\mathrm{kW}_{\mathrm{p}}\right]}\end{array}$ & $\begin{array}{c}\text { Battery } \\
\text { size } \\
{[\mathbf{k W h}]}\end{array}$ & $\begin{array}{c}\text { PV panels } \\
\text { cost } \\
{[€]}\end{array}$ & $\begin{array}{c}\text { Converter } \\
\text { cost } \\
{[€]}\end{array}$ & $\begin{array}{c}\text { Fixtures } \\
\text { and labour } \\
{[€]}\end{array}$ & $\begin{array}{c}\text { Battery } \\
\text { cost } \\
{[€]}\end{array}$ & $\begin{array}{c}\text { Total } \\
\text { Costs } \\
{[€]}\end{array}$ & $\begin{array}{c}\text { Annualized } \\
\text { total costs } \\
\text { [€/year] }\end{array}$ & $\begin{array}{l}\text { Weighed } \\
\text { Lifespa } \\
\text { [years] }\end{array}$ & $\begin{array}{l}\text { Yearly } \\
\text { Savings } \\
\text { [€/year] }\end{array}$ & $\begin{array}{c}\text { Payback } \\
\text { period } \\
\text { [years] }\end{array}$ & $\begin{array}{c}\text { Global } \\
\text { yearly } \\
\text { savings }[€]\end{array}$ \\
\hline \multirow{3}{*}{$\begin{array}{c}\text { Siz. } \\
\text { I }\end{array}$} & Bill. 1 & \multirow{3}{*}{4.5} & \multirow{3}{*}{11.2} & \multirow{3}{*}{1905.80} & \multirow{3}{*}{2550.80} & \multirow{3}{*}{4407.90} & \multirow{3}{*}{5975.50} & \multirow{3}{*}{14840.00} & \multirow{3}{*}{911.52} & \multirow{3}{*}{16.3} & 623.80 & 23.8 & -287.72 \\
\hline & Bill. 2 & & & & & & & & & & 642.75 & 23.1 & -268.77 \\
\hline & Bill. 3 & & & & & & & & & & 657.14 & 22.6 & -254.38 \\
\hline \multirow{3}{*}{$\begin{array}{c}\text { Siz. } \\
\text { II }\end{array}$} & Bill. 1 & \multirow{3}{*}{3.4} & \multirow{3}{*}{2.8} & \multirow{3}{*}{1439.90} & \multirow{3}{*}{2455.20} & \multirow{3}{*}{4217.70} & \multirow{3}{*}{2759.80} & \multirow{3}{*}{10873.00} & \multirow{3}{*}{561.78} & \multirow{3}{*}{19.4} & 540.32 & 20.1 & -21.46 \\
\hline & Bill. 2 & & & & & & & & & & 584.62 & 18.6 & 22.83 \\
\hline & Bill. 3 & & & & & & & & & & 616.92 & 17.6 & 55.13 \\
\hline \multirow{3}{*}{$\begin{array}{l}\text { Siz. } \\
\text { III }\end{array}$} & Bill. 1 & \multirow{3}{*}{4.5} & \multirow{3}{*}{0.0} & \multirow{3}{*}{1905.80} & \multirow{3}{*}{2560.00} & \multirow{3}{*}{4407.90} & \multirow{3}{*}{0.00} & \multirow{3}{*}{8873.60} & \multirow{3}{*}{314.43} & \multirow{3}{*}{28.2} & 410.18 & 21.6 & 95.76 \\
\hline & Bill. 2 & & & & & & & & & & 571.37 & 15.5 & 256.94 \\
\hline & Bill. 3 & & & & & & & & & & 662.25 & 13.4 & 347.83 \\
\hline
\end{tabular}




\section{CONCLUSIONS}

As it has been shown, in the analyzed cases savings via self-consumption range from $40 \%$ and can be as high as $70 \%$ of the electricity bill. However, global savings, considering the investment costs, highly varies.

This is not only due to the sizing and billing method, but it has been also shown that it depends on other factors such as consumption level or power profile. This is in turn caused by high fixed prices and the importance of instantaneous selfconsumption, which is always preferable to storing.

Nevertheless, in all cases, the self-consumption system is already profitable in the absence of a battery. However, even in this case, correct sizing is crucial for profitability, thus the need of a stable regulation framework especially in a sector where payback periods are usually higher than in other activities.

Most importantly, the profitability of the systems with battery has been seen to be mainly conditioned by the battery cost and its lifespan, which combined make the annualized cost of the battery to be between 50 and $63 \%$ of the total annualized cost in the analyzed cases. Nevertheless, the cost and lifespan of the batteries are expected to decrease and increase respectively, leading to a promising future in the selfconsumption sector. This is a critical aspect as net metering is a viable solution for certain systems in a low penetration level of renewables scenario, however, in the long run, in a high penetration level of renewables scenario, batteries along with the appropriate energy management strategy will be needed for energy management. Therefore, subsidizing solar battery systems now, that are near to be profitable, seems a good option as it will boost the adoption and, hence the technological advance, of batteries.

\section{REFERENCES}

[1] Protocolo de Kioto CMNUCC 1998. [Online]. Available: https://goo.gl/J3Pivd. [Accessed: 22-Jan-2018]

[2] Conferencia de las Partes UNFCCC 2015-12-11, relativa a la Aprobación del Acuerdo de París. [Online]. Available: https://goo.gl/HZ5dbn. [Accessed: 23-Jan-2018]

[3] Directiva del Parlamento Europeo y del Consejo por la que se modifica la Directiva 2012/27/UE, relativa a la eficiencia energética (Paquete de Invierno). [Online]. Available: https://goo.gl/dSvpcG. [Accessed: 24Jan-2018]

[4] Directiva (UE) 2015/1513 del Parlamento Europeo y del Consejo de 9 de septiembre de $2015, \ldots$, relativa al fomento del uso de energía procedente de fuentes renovables. [Online]. Available: https://goo.gl/2FC9q7. [Accessed: 23-Jan-2018]

[5] «Plan de Energías Renovables (PER) 2011-2020», IDAE, Madrid, España, 2011 [Online]. Available: https://goo.gl/ZY1hjt. [Accessed: 23-Jan-2018]

[6] «Resumen Plan de Energías Renovables (PER) 2011-2020», IDAE, Madrid, España, jul. 2011 [Online]. Available: https://goo.gl/EqgpKb. [Accessed: 23-Jan-2018]

[7] «Plan de Acción de Ahorro y Eficiencia Energética (NEEAP) 20112020», IDAE, Madrid, España [Online]. Available: https://goo.gl/DChJVC. [Accessed: 23-Jan2018]

[8] «Resumen del Plan de Acción de Ahorro y Eficiencia Energética (NEEAP) 2011-2020», IDAE, Madrid, España, jul. 2011 [Online]. Available: https://goo.gl/WCV2Vr. [Accessed: 23-Jan-2018]

[9] «Plan Nacional de Acción de Eficiencia Energética 2017-2020», IDAE, Madrid, España, may 2017 [Online]. Available: https://goo.gl/iG7Kvu. [Accessed: 23-Jan-2018]

[10] «PV Grid Parity Monitor», CREARA, Madrid, España, may 2018 [Online]. Available: https://goo.gl/hcn7E8. [Accessed: 27-Feb-2019]
[11] R. Luthander, J. Widén, D. Nilsson, y J. Palm, «Photovoltaic selfconsumption in buildings: A review», Applied Energy, vol. 142, pp. 80-94, 2015 [Online]. Available: https://goo.gl/Q6nQgs. [Accessed: 18-Jan-2018]

[12] J. López Prol y K. W. Steininger, «Photovoltaic self-consumption regulation in Spain: Profitability analysis and alternative regulation schemes», Energy Policy, vol. 108, pp. 742-754, 2017 [Online]. Available: https://goo.gl/2H6Kro. [Accessed: 18-Jan-2018]

[13] V. Aragonés, J. Barquín, y J. Alba, «The New Spanish Selfconsumption Regulation», en Energy Economics Iberian Conference, EEIC 2016, 4-5 February 2016, Lisbon, Portugal, 2016, vol. 106, pp. 245-257 [Online]. Available: https://goo.gl/gBnBnT. [Accessed: 18Jan-2018]

[14] F. J. Ramírez, A. Honrubia-Escribano, E. Gómez-Lázaro, y D. T. Pham, «Combining feed-in tariffs and net-metering schemes to balance development in adoption of photovoltaic energy: Comparative economic assessment and policy implications for European countries», Energy Policy, vol. 102, pp. 440-452, 2017 [Online]. Available: https://goo.gl/QBgg8o. [Accessed: 18-Jan-2018]

[15] R. Dufo-López y J. L. Bernal-Agustín, «A comparative assessment of net metering and net billing policies. Study cases for Spain», Energy, vol. 84, pp. 684-694, 2015 [Online línea]. Available: https://goo.gl/Rsavig. [Accessed: 18-Jan-2018]

[16] C. A.-T. M. Díez-Mediavilla, E. Bueno-Rastrilla, M. C. RodríguezAmigo, M. de Simón-Martín, «Net Energy Metering: A case study in Spain», PVSEC'14, vol. 1, 2015 [Online]. Available: https://goo.gl/2a7FDG. [Accessed: 16-Feb-2019]

[17] V. Q. Johannes Weniger, Joseph Bergner, Tjarko Tjaden, «Economics of Residential PV Battery Systems in the Self-Consumption Age», PVSEC, sep. 2014 [Online]. Available: https://goo.gl/1nX5ta. [Accessed: 16-Feb-2019]

[18] G. Cerino Abdin y M. Noussan, «Electricity storage compared to net metering in residential PV applications», Journal of Cleaner Production, vol. 176, p. 175, 2018 [Online]. Available: https://goo.gl/x1ydhc. [Accessed: 18-Jan-2018]

[19] «Análisis del consumo energético del sector residencial en España», IDAE, España, jul. 2011 [Online]. Available: https://goo.gl/bVwvGD. [Accessed: 23-Jun-2018]

[20] Real Decreto 244/2019, de 5 de abril, por el que se regulan las condiciones administrativas, técnicas y económicas del autoconsumo de energía eléctrica. [Online]. Available: https://bit.ly/2vnrwhd. [Accessed: 8-Apr-2019] 